\title{
Modelo pedagógico y estrategias de implementación académica, de investigación y de vinculación para la formación ambiental en la licenciatura en educación inicial
}

\author{
Rufina E. Mejía ${ }^{1}$, Milagro C. Rodríguez ${ }^{1 *}$, Tania Merino ${ }^{3}$, y Teresa Torres ${ }^{4}$ \\ (1) Unidad Académica de Educación, Artes y Humanidades, Sede Macas. Universidad Católica de Cuenca, Ecuador \\ (Correo-e: rmejiap@ucacue.edu.ec, mrodrigueza@ucacue.edu.ec) \\ (2) Ministerio de Educación Superior, La Habana, Cuba (Correo-e: taniam@mes.gob.cu) \\ (3) Centro de Estudios de Perfeccionamiento de la Educación Superior, Universidad de la Habana, Cuba \\ (Correo-e: torresmiranda@cepes.uh.cu)
}

${ }^{*}$ Autor a quien debe ser dirigida la correspondencia.

Recibido Jun. 25, 2021; Aceptado Ago. 24, 2021; Versión final Oct. 6, 2021, Publicado Feb. 2022

\begin{abstract}
Resumen
El objetivo principal de este estudio fue valorar un modelo pedagógico y su estrategia de implementación para la formación ambiental en la licenciatura en educación inicial. Se integra lo académico, la investigación y la vinculación. La metodología fue descriptiva explicativa, con enfoque mixto, desarrollada en tres fases. Se utilizaron encuestas, entrevistas, grupos focales y un pre-experimento. El muestreo, no probabilístico intencional, incluyó 20 estudiantes, siete profesores de la carrera, 30 profesores de otras carreras, cinco directivos y 24 educadores parvularios. Los resultados revelaron una transformación positiva de la formación ambiental de los estudiantes en sus dimensiones cognoscitiva, procedimental y actitudinal. Se observó en el registro de incidencia que cinco indicadores tendieron hacia el nivel medio y tres al nivel alto. El preexperimento mostró que un $90 \%$ de los alumnos logró transitar a un nivel superior. En conclusión, es posible promover la formación ambiental desde la universidad con acciones que integren actividades que relacionen la docencia, la investigación y la vinculación.
\end{abstract}

Palabras clave: dimensión ambiental; docencia; estrategia; investigación; modelo pedagógico; sociedad

\section{Pedagogical model and academic, research, and enrollment implementation strategies for environmental training in infant education degrees}

\begin{abstract}
The primary objective of this study was to assess a pedagogical model and its implementation strategy for environmental training in an infant education bachelor's degree. The strategy integrates academic, research, and enrollment aspects. The methodology was descriptive-explanatory, with a mixed approach, and was developed in three phases. Surveys, interviews, focus groups, and a pre-experiment were applied. The sampling was intentional non-probabilistic and included 20 students, seven infant education bachelor's degree professors, 30 professors from other degree programs, five managers, and 24 kindergarten educators. The results showed a positive transformation in students' environmental training in its cognitive, procedural, and attitudinal dimensions. The incidence record revealed that five indicators were at a medium level and that three indicators were at a high level. The pre-experiment showed that $90 \%$ of the students managed to move to a higher level. In conclusion, it is feasible to promote environmental training in universities with initiatives that integrate activities that connect teaching, research, and outreach.
\end{abstract}




\section{INTRODUCCIÓN}

La aguda situación ambiental que enfrenta la humanidad, afecta cada año mayores áreas del planeta, por ende, se hace inaplazable repensar el comportamiento humano y sentar las bases de un futuro sostenible. A este hecho se asocia el surgimiento y desarrollo de la educación ambiental, la que según Flores (2010), por su magnitud y dimensiones actuales, conlleva a la necesidad de interpretarla desde una visión holísticointegradora que explique las bases de la crisis ambiental. Desde las primeras reuniones internacionales promovidas por la UNESCO, como la Conferencia Mundial sobre el Medio Ambiente, en Estocolmo Suecia, 1972, se propicia la reflexión acerca del medio ambiente y proveer alternativas de solución a su problemática. Luego de la Conferencia Intergubernamental sobre Educación Ambiental en Tbilisi, Georgia, 1977, los países latinoamericanos comienzan a incorporar paulatinamente en sus programas educativos material referente al medio ambiente, su cuidado y protección; los que, luego de aprobada la Agenda 21 en Río de Janeiro, 1992, incorporan criterios de sostenibilidad.

Las metas de los 17 Objetivos de Desarrollo Sostenible (ODS), valorados como una agenda transformadora e inclusiva, llaman a cambiar el estilo de desarrollo respetando el medio ambiente; constituyen hoy, el compromiso universal adquirido por la mayoría de los países en el marco de una alianza mundial reforzada, que toma en cuenta los medios de implementación para el cambio y la reducción de riesgos de desastres con enfoque de mitigación y adaptación al cambio climático, (CEPAL, 2016). Lo anterior refuerza la preocupación de actores sociales sobre los problemas ambientales y a la vez, la necesidad de establecer propuestas de formación ambiental holístico-integradora, que impacten en la calidad de la formación profesional, fundamentalmente en la de profesores. La universidad constituye un escenario privilegiado para diseñar propuestas pedagógicas que contribuyan a la formación ambiental y faciliten la implementación de una educación para el desarrollo Sostenible, considerando que los estudiantes a futuro ocuparan puestos profesionales en distintos espacios laborales, constituyendo un motor de cambio para responder a los nuevos desafíos ambientales (Alcalá-Del-Olmo-Fernández et al., 2021).

En el contexto ecuatoriano, la formación ambiental se fortalece en el marco normativo desde la Constitución Política de la República del Ecuador, la Ley Orgánica de Educación Intercultural y la Ley Orgánica de Educación Superior (LOES), que se hace eco de la política en torno a la formación ambiental al pretender "fomentar y ejecutar programas de investigación de carácter científico, tecnológico y pedagógico que coadyuven al mejoramiento y protección del ambiente y promuevan el desarrollo sustentable nacional" (Asamblea Nacional del Ecuador, 2018). Derivado de los mandatos políticos y en respuesta a la problemática ambiental del país; se han podido identificar resultados de investigaciones sobre el tema, se destacan los aportes de Zambrano (2015) al enfoque de la educación para el desarrollo sostenible en la formación docente, quien aseveran la existencia de un desconocimiento sobre la educación ambiental por una parte significativa de actores de la educación, destaca la necesidad de la capacitación de docentes y la incorporación de la educación ambiental en los currículos y su abordaje en el espacio educativo.

En la actualidad, algunos autores como (González, 2006; Mejía, 2016; Ardoin et al., 2020) consideran a la educación una vía eficaz para transformar la problemática ambiental actual, desarrollar sentimientos, actitudes y valores necesarias para la conciliación entre medio ambiente y desarrollo; y evidencian la necesidad de construir escenarios de aprendizaje donde, los profesores en formación gestionen el conocimiento ambiental desde la investigación y la vinculación con la sociedad, asociado al desarrollo sostenible y el compromiso generacional. Otros, por su parte, analizan cómo la formación ambiental en Latinoamérica y particularmente en la Región Sur del Ecuador enfrenta varias problemáticas, entre ellas, el inadecuado perfil docente que impone cambios inter y transdisciplinarios en los modelos pedagógicos de modo que, incidan en la actitud y el comportamiento de las autoridades educativas y del propio docente, en el medio ambiente unido al bienestar humano (Isch y Zambrano, 2008).

De igual modo De-la-peña y Vince (2019) alertan sobre la poca disponibilidad de investigaciones relacionadas con la educación ambiental en el contexto universitario del Ecuador, profundizan en la necesidad de atender su incorporación en la formación inicial de profesionales, y aportan propuestas valiosas de obligada consulta. A partir del criterio anterior, y contextualizando a la formación ambiental de estudiantes que se preparan como futuros profesionales de la Educación Inicial, se resalta la importancia de incorporar la dimensión ambiental en el proceso formativo desde sus componentes docencia, investigación y de vinculación con la sociedad.

En la Universidad Católica de Cuenca, el Proyecto de Diseño de la Carrera de Educación Inicial, expresa, la necesidad de elaborar estrategias que propicien, entre otros objetivos, la convivencia armónica con la comunidad y la naturaleza. Sin embargo, en los estudiantes de la carrera de educación inicial, se manifestaban carencias cognoscitivas y procedimentales que se reflejaban en lo actitudinal e impedían la comprensión y tratamiento de los problemas ambientales con un enfoque interdisciplinar. De ahí que el problema fundamental a debatir fue, ¿cómo integrar la formación ambiental en la formación profesional del estudiante de Licenciatura en Educación Inicial en la Universidad Católica de Cuencas, sede Macas? El 
objetivo de esta disertación se encamina a valorar un modelo pedagógico y su estrategia de implementación que integran lo académico, la investigación y la vinculación para la formación ambiental en la Licenciatura en Educación Inicial.

\section{METODOLOGÍA}

La investigación fue de tipo descriptiva explicativa, con un enfoque mixto cuali-cuantitativo, se aplicaron métodos teóricos, empíricos y matemáticos estadísticos en el desarrollo de sus tres fases para cumplir con el objetivo establecido. Se realizó un muestreo no probabilístico intencional, tomando un grupo de estudio integrado por 20 estudiantes de sexto ciclo, el criterio de selección estuvo asociado a que este grupo desarrollaría las actividades de la práctica preprofesional y además recibirían asignaturas que estarían implicadas en la implementación parcial de la estrategia. Participaron, además los 7 docentes de la Licenciatura en Educación Inicial, 24 docentes de Educación Inicial de las diferentes instituciones educativas en las que se realizaron las prácticas pre-profesionales, 23 docentes de otras carreras y 5 directivos para la participación en los talleres. Todos dieron su consentimiento por escrito para participar en la investigación.

La primera fase fue realizar una revisión bibliográfica utilizando el análisis documental basado en los siguientes criterios: 1) búsqueda de artículos, libros, capítulos de libros y otros documentos en idioma español e inglés en las principales bases de datos, Scielo, Scopus, Web of Science, Redalyc, Google Académico para lo cual se utilizaron descriptores de búsqueda con la combinación de términos como: formación ambiental, dimensión ambiental, formación profesional, educación superior; 2) período de análisis 2010-2020; la información seleccionada se organizó en el procesador bibliográfico Mendeley, lo que permitió realizar un estudio para identificar las principales definiciones de formación ambiental y las dimensiones e indicadores para su medición, las cuales se presentan en la sección de resultados como insumo obtenido en esta fase.

La segunda fase comenzó caracterizando el estado inicial de la variable "formación ambiental" en los estudiantes de la Licenciatura en Educación Inicial de la Sede Macas de la Universidad Católica de Cuenca, para lo cual se aplicó una encuesta, una entrevista grupal a los alumnos cuyos ítems respondieron a las dimensiones cognitiva, procedimental y actitudinal y una guía de observación a los estudiantes (valoración de la dimensión procedimental y actitudinal), además se aplicó la entrevista a los profesores de la carrera con la finalidad de conocer la percepción de los docentes sobre la formación ambiental de los estudiantes. Los instrumentos utilizados fueron validados a través de una prueba piloto y el método de criterio de experto obteniendo en general una valoración de bastante adecuados para el propósito de la investigación.

Para el análisis e interpretación de los resultados del diagnóstico inicial, se utilizó la triangulación metodológica concebida como la combinación de diferentes métodos con el objetivo de obtener una imagen más adecuada y completa del objeto de estudio de la investigación (Kelle y Bernhard, 2019). Lo que facilito la obtención de resultados más completos con la confrontación de los hallazgos obtenidos derivados de la encuesta, la entrevista grupal y la guía de observación a los estudiantes, así como de la entrevista a los profesores. Partiendo de los resultados aportados por la revisión bibliográfica y del diagnóstico realizado para obtener la caracterización inicial de la variable se procedió en segunda instancia a la construcción del modelo pedagógico y la estrategia para su implementación. En este proceso se aplicaron métodos teóricos como el análisis y la síntesis, la modelación y el sistémico estructural.

La tercera fase implicó la implementación de la estrategia como instrumento práctico del modelo y además la evaluación de los resultados de su puesta en práctica. La implementación parcial se realizó en la Unidad Académica de Educación, en la Sede Macas de la Universidad Católica de Cuenca, durante la misma, se diseñó una intervención gradual en la práctica educativa con el objetivo de comprobar la trasformación en la formación ambiental de los estudiantes mediante acciones preconcebidas para tres momentos: 1er momento de coordinación y exploración; 2do momento de desarrollo; 3er momento de medición y evaluación de los resultados de la implementación parcial de la estrategia.

\section{Procedimiento empleado}

1er Momento de coordinación y exploración: Su objetivo fue el de coordinar y explorar con los directivos de la unidad académica, de la sede y de la carrera; los espacios y oportunidades más factibles para la implementación del modelo pedagógico mediante su estrategia. Los talleres de reflexión que se describen a continuación, formaron parte de este momento seguidos de reuniones metodológicas desarrolladas con los profesores. Los Talleres de Reflexión Crítica que se desarrollaron, tuvieron como finalidad la de socializar la caracterización de la formación ambiental de los estudiantes, obtener mayor información al respecto y valorar la factibilidad de la estrategia propuesta como vía para la implementación del modelo pedagógico. Se necesitó del criterio valorativo de los docentes en cuanto a la aplicabilidad de la estrategia en el contexto de la Educación Superior ecuatoriana y su correspondencia con las características de los estudiantes universitarios. 
Los talleres se ejecutaron empleando la técnica del focus group; primeramente, se procedió a su diseño metodológico lo que tomó en cuenta la cantidad de participantes, la carrera en que trabajan, la experiencia profesional en la Educación Superior y además los elementos teóricos y práctico-metodológicos a tratar. Los grupos creados fueron multidisciplinarios. Las sesiones de trabajo concebidas, quedaron conformadas como se describe a continuación:

Taller 1. Presentación de la investigación y sensibilización de docentes de la Licenciatura en Educación Inicial. Participaron tres directivos (el Coordinador de la sede Macas, la Directora del Departamento de Investigación y la Directora de carrera), unido a siete docentes de Educación Inicial. Su objetivo fue el de sensibilizar a los participantes en el proceso de formación ambiental de los estudiantes de la Licenciatura en Educación Inicial.

Taller 2. Presentación del modelo pedagógico y su estrategia de implementación en la sede Macas. Participaron 30 docentes de las carreras Licenciatura en Educación Inicial, Enfermería, Contabilidad y Administración de Empresa, como objetivo se propuso el socializar entre la comunidad académica de la sede Macas, las ideas esenciales de un modelo pedagógico para la formación ambiental de los estudiantes de la Licenciatura en Educación Inicial y su estrategia de implementación.

Taller 3. Presentación del resultado científico, bases teórico-metodológicas y necesidad de la investigación. Participaron directivos y docentes de la carrera de Educación Inicial en la sede Macas (8); especialistas en el tema ambiental de otras sedes de la UCACUE (3); Directiva de la Unidad Académica (Decana); delegado del Ministerio del Agua y del Ambiente de la zonal 6 en la provincia Morona-Santiago; tutoras de tesis (2). El objetivo central radicó en compartir con la comunidad académica y científica los sustentos de la investigación y el resultado científico aportado. La sesión se desarrolló vía on-line mediante la plataforma Zoom.

2do Momento de desarrollo: su objetivo consistió en ejecutar las actividades concebidas desde la etapa de planificación de la estrategia, las que incluyen la capacitación a los docentes y las actividades a desarrollar con los estudiantes. Sobre la capacitación a docentes, se empleó el método de entrenamiento metodológico desde el puesto de trabajo y a distancia. Con respecto a los estudiantes se emplearon métodos como la investigación acción desarrollando un total de 12 actividades, desde la práctica pre-profesional en el período evaluado (septiembre/2019 - febrero/2020) y la cátedra de Necesidades Educativas Especiales (marzo agosto/2020).

3er Momento de medición y evaluación de los resultados de la implementación parcial de la estrategia: su objetivo fue valorar la formación ambiental lograda en los estudiantes a partir de los resultados alcanzados en las acciones ejecutadas en los componentes de docencia, investigación y vinculación. La medición y evaluación de los resultados obtenidos, se realizó en una primera instancia con un carácter procesal mediante un registro de incidencias para evaluar la variable por sus indicadores en niveles bajo, medio y alto; a cada uno de ellos se les colocó un valor utilizando una escala de bajo=0; medio=1; alto= 2 .

También se aplicó el método experimental con un diseño preexperimental en el que participaron los 20 alumnos de 6 to ciclo que formaron parte de la muestra. El preexperimento se concibió con un grupo único con el esquema de aplicación de una prueba pedagógica de entrada al inicio del período analizado (septiembre 2019), posteriormente se realizó la intervención con la aplicación del modelo mediante el desarrollo de las actividades planificadas en la estrategia y al final (agosto 2020) se realizó una prueba pedagógica de salida. Ambas pruebas fueron elaboradas con preguntas que permitieron medir las dimensiones e indicadores definidos para la variable objeto de estudio "Formación Ambiental".

Se utilizó la Prueba Estadística de Rangos con signos de Wilcoxon para dos muestras relacionadas de modo que permitiera demostrar la validez de la hipótesis estadística planteada en esta etapa de la Investigación. Para ello se recolectaron los datos de la prueba de entrada y la prueba de salida de los 20 estudiante y se sometió la variable a un análisis del antes y el después, (Triola, 2009). Para el estudio se partió del siguiente par de hipótesis estadísticas: i) Ho (hipótesis nula): No hay diferencias significativas entre los puntajes relativos a las pruebas de rendimiento que miden las dimensiones e indicadores de la formación ambiental de los estudiantes antes y después de la aplicación del modelo pedagógico mediante la estrategia; y ii) $H 1$ (hipótesis alternativa): Los puntajes relativos a las pruebas de rendimiento que miden las dimensiones e indicadores de la formación ambiental de los estudiantes antes y después de la aplicación del modelo pedagógico mediante la estrategia, sí difieren significativamente. Se adoptó un nivel de significación ( $\propto$ ) de 0,05 para los tratamientos estadísticos.

\section{RESULTADOS Y DISCUSIÓN}

Seguidamente se detallan los resultados más significativos obtenidos en cada una de las tres fases de la investigación, realizando énfasis en aquellos que se obtuvieron en la fase tres correspondiente a la implementación parcial de la estrategia. 


\section{Primera fase de la Investigación}

Después de la revisión bibliográfica realizada, a los efectos de este estudio se definió a la formación ambiental como el proceso gradual, permanente holístico e integrador que se da en todos los espacios y componentes de la formación universitaria donde, a partir de la gestión del conocimiento, la investigación y la participación en cuestiones asociada al medioambiente; se modelan procedimientos y actitudes que regulan los modos de actuación del futuro docente de la Educación Inicial; al tiempo que se sistematizan buenas prácticas medioambientales. Su consideración como dimensión de la formación profesional de los estudiantes (Merino, 2019), se fundamentó desde los referentes del constructivismo social y la pedagogía crítica que, con enfoque holístico e integrador, se trabajó desde la docencia, la investigación y la vinculación con la sociedad. Para su estudio y caracterización, se le clasificó como variable compleja, (Carballo y Guelmes, 2016), lo que requirió la determinación de dimensiones a manera de niveles de análisis intermedio entre esta y los indicadores.

La precisión definitiva de cuáles dimensiones considerar y mediante qué indicadores evaluarlas, surgió de la sistematización de criterios de varios autores quienes investigaron este objeto en contextos diferentes. Los elementos considerados en el estudio fueron los siguientes: relaciones con el problema científico y objetivo de la investigación (Mejías et al., 2018); (Cóndor, 2018); correspondencia con las posiciones teóricas asumidas (Boca y Saraçli, 2019); (Álvarez et al., 2015), posibilidades reales de análisis en la situación concreta que se investiga (Grishaeva et al., 2018); (Davis, 2020). Como resultado de la sistematización realizada, se determinó que, para su estudio la formación ambiental de estudiantes de la carrera Licenciatura en Educación Inicial, se descompusiera en tres dimensiones que permitieron su valoración y análisis desde el estado inicial al final; ellas fueron:

Dimensión cognitiva: abarca el sistema de conocimientos (conceptos, categorías, relaciones) ambientales y pedagógicos necesarios de gestionar para la formación ambiental de los estudiantes, centrado en la interpretación del medio ambiente como sistema, la argumentación de la problemática ambiental, sus causas y consecuencias; la valoración de la formación ambiental como la base para desarrollar procesos educativo ambientales con niños de la primera infancia y el conocimiento de la legalidad ambiental vigente.

Dimensión procedimental: Hace referencia al modo de actuación e incluye las habilidades profesionales y las competencias investigativas necesarias para que los estudiantes en su desempeño profesional futuro desarrollen procedimientos y metodologías que le permitan detectar problemas asociados a la educación ambiental en la primera infancia, emitan juicios valorativos y formen parte de la acción en la búsqueda de la solución a los problemas detectados.

Dimensión actitudinal: comprende las actuaciones frente a sí mismo, su entorno natural y social, en función de conocer, sentir y actuar por el medio ambiente; puede modelar comportamientos en la medida que esta sea desarrollada. Los comportamientos se evalúan en forma permanente y se expresan en forma de actitudes, los valores y las expresiones. Los indicadores definidos para su medición fueron: muestra sensibilidad ante los problemas ambientales y sus causas; disposición para actuar en prácticas ambientales sostenibles y sistematización de acciones y buenas prácticas en el contexto educativo.

\section{Segunda fase de la Investigación}

Para la caracterización del estado inicial de la variable se fueron aplicando los instrumentos según los métodos establecidos en esta fase de la investigación y señalados en la sección de la metodología. Los resultados al utilizar la triangulación metodológica evidenciaron que: en la dimensión cognitiva existía la necesidad de una mayor preparación teórica de contenidos medioambientales y preparación de los profesores en temas que contribuyan a la formación ambiental; en la dimensión procedimental se detectaron deficiencias en el desarrollo de las habilidades para aplicar los conocimientos en la práctica, a través de procedimientos y metodologías asociadas a la educación ambiental. Estos resultados de la caracterización coinciden en algunos puntos con los obtenidos en el estudio de (Álvarez et al., 2019) donde se realizó un análisis de las variables que influyen en la formación inicial del profesorado en educación para el desarrollo sostenible. Al profundizar en la dimensión actitudinal de los estudiantes que formaron parte de la investigación, se detectó que estos no estaban sensibilizados con considerar al ser humano como sujeto de derecho; ni en la importancia de introducir buenas prácticas ambientales en el proceso educativo.

La construcción del modelo pedagógico y su estrategia de implementación se apoyó en los supuestos de orden filosófico, sociológico, psicológico, epistemológicos y pedagógicos expuestos en el modelo educativo de la Universidad Católica de Cuenca (UCACUE, 2016). Entre los rasgos que distinguen el modelo están el énfasis en los componentes personales del proceso pedagógico de la formación ambiental con métodos dinámicos, flexibles, participativos y no directivos; sin desatender la unidad indisoluble, con los componentes no personales en la docencia, la investigación y la vinculación con la sociedad; y el involucramiento de profesores y estudiantes, en la gestión del conocimiento ambiental, durante la cual asumieron un rol activo, participativo, reflexivo, investigativo y experimentador, elementos que coinciden con los planteados por (Cuenca et al., 2021) en su investigación. 
La novedad del modelo propuesto respecto a otros consultados en la literatura como el de (Mejías et al., 2018; Martínez-Iñiguez et al., 2021) radica en la asunción de una concepción holístico integradora que, tanto en el plano teórico como práctico, permitió analizar la formación ambiental de los estudiantes de la Licenciatura en Educación Inicial desde sus dimensiones cognitiva, procedimental y actitudinal a través de la integración de los procesos: docencia, investigación y vinculación con un enfoque interdisciplinar. Su estructuración, atendiendo a la complejidad del objeto investigado, consideró la teoría general de los sistemas de Bertalanffy (1969), por sus criterios de totalidad en tanto los elementos que lo componen, a pesar de poseer propiedades determinadas y cumplir funciones específicas, constituyen una unidad dialéctica, de la cual emergen propiedades cualitativamente superiores como síntesis de las relaciones entre ellos, lo cual caracteriza su concepción y desarrollo. Los componentes que lo integraron son:

Subproceso académico de la formación ambiental: se caracterizó por el desarrollo de ambientes de aprendizaje que incorporaron actividades pedagógico-ambientales orientadas a la contextualización, organización, explicación y sistematización del conocimiento científico, técnico y profesional necesario para que el estudiante se apropiara de los procedimientos y actitudes que le permitan planificar y proyectar procesos de educación ambiental en sus contextos de actuación profesional y ciudadana con alto valor humanístico.

Subproceso investigativo de la formación ambiental: la organización de la actividad científica asociada a la formación ambiental de los estudiantes, se sustentó en los requerimientos del proceso de investigación en el nivel de grado, se ponderó el desarrollo de conocimientos y actitudes para la innovación científica, tecnológica, humanística y ética, conforme elementos formativos de la epistemología y la metodología de investigación asociada a la profesión, mediante el desarrollo de proyectos de investigación ambiental de carácter exploratorio y descriptivo. Incluyó la atención al desarrollo de habilidades investigativas que permitieron al estudiante desarrollar una actitud científica respecto a la problemática ambiental en sus contextos de actuación profesional y ciudadana.

Subproceso vinculación con la sociedad para la formación ambiental: Incluyó las actividades o programas que articularon la formación ambiental de los estudiantes con el régimen constitucional del Buen Vivir, el Plan Nacional de Desarrollo, las necesidades del desarrollo local, regional y nacional, los requerimientos sociales de su área de influencia y las corrientes internacionales científicas y humanísticas de pensamiento. Fue este, el subsistema integrador entre la formación profesional que opera en la universidad y los requerimientos que, en materia de formación ambiental, debe poseer el estudiante para interactuar en su entorno.

Para la implementación en la práctica educativa, del modelo pedagógico se definieron los pasos contenidos en una estrategia, estructurada en acciones que permitieron evaluar la transformación del proceso de formación ambiental de los estudiantes, a partir de los objetivos específicos siguientes: habilitar a los docentes de herramientas metodológicas para el perfeccionamiento del proceso de formación ambiental que ha de desarrollarse en la universidad en cumplimiento de sus estatutos; promover procedimientos y actitudes reflexivas en los estudiantes en torno al medioambiente mediante la gestión de contenidos pedagógicos y ambientales ajustados a la educación inicial; desarrollar en los estudiantes modos de actuación dirigidos a la realización de acciones educativo-ambiental con los infantes y sus familias, como reflejo de la formación ambiental alcanzada, aspectos que son coincidentes con los señalados por algunos autores como (Sudan y Zuin, 2018) y (Grishaeva et al., 2018) quienes destacaron esencialmente la necesidad de trabajar desde la universidad a partir del trabajo metodológico de los docentes para desarrollar el pensamiento crítico en los estudiantes en el proceso de educación ambiental, así como el accionar pedagógico en la formación de la experiencia reflexiva de los alumnos en relación a los problema de la "localidad" y la "focalización" de los contenidos y métodos de la educación para el desarrollo sostenible desde enfoques inter y transdisciplinarios.

La estrategia de implementación se distinguió por su carácter integrador de la formación ambiental, el análisis del sistema de conocimiento de las diferentes asignaturas de la carrera relacionadas con la formación ambiental, la determinación de los núcleos temáticos y de contenidos que tributaron a sus objetivos; así como las acciones y operaciones coordinadas que permitieron su ejecución. Fue compuesta por tres etapas. La primera etapa de la estrategia, denominada preparatoria y diagnóstica del estado de la formación ambiental de los estudiantes, se concibió con la finalidad de preparar a los profesores de la carrera y a los estudiantes para desarrollar las acciones previstas, tomó en cuenta el diagnóstico del estado de desarrollo de la formación ambiental de los estudiantes, potencialidades y necesidades; y el entrenamiento metodológico en temas de formación ambiental para docentes de la UCACUE.

La segunda etapa de la estrategia, denominada de planificación y ejecución, se concibió con el propósito de desarrollar actividades que propiciaran la formación ambiental de los estudiantes en los diferentes ciclos en la UCACUE, para su desarrollo se estableció: la preparación técnica, didáctica y metodológica de los profesores de la carrera de Educación Inicial y; la estructuración del trabajo metodológico de modo que permitiera la integración de los componentes docencia, investigación y vinculación. Para ello, la planificación requirió de actividades de formación ambiental que consideraran tanto, los objetivos formativos del modelo 
educativo de la UCACUE, como los resultados del diagnóstico aplicado en la etapa anterior. Las formas organizativas estimadas fueron: actividades docentes, las tareas de la práctica de experimentación y aprendizaje, el trabajo autónomo y los proyectos integradores, en su vínculo con los contenidos relacionados con la formación ambiental. La tercera y última etapa de la estrategia, concebida para la evaluación de la transformación e impactos alcanzados, se previó con la finalidad de valorar la formación ambiental lograda en los estudiantes a partir de su propia autovaloración; de los resultados alcanzados en las acciones ejecutadas en los componentes de docencia, investigación y vinculación; y de la medición de los resultados alcanzados en el centro de prácticas preprofesionales.

\section{Tercera fase de la investigación}

El intercambio permanente de los que participaron en el proceso, permitió evaluar la pertinencia del modelo pedagógico, así como de la estrategia diseñada para su implementación en la práctica educativa. Para el intercambio y enriquecimiento del resultado científico fueron utilizados los tres talleres mencionados en la metodología, mediante los cuales se recibieron contribuciones críticas. El trabajo metodológico se formuló en términos de demostrar a los docentes, cómo organizar el proceso de formación ambiental en la carrera de Licenciatura en Educación Inicial. En lo adelante se ofrece una descripción de los resultados de los talleres de reflexión desarrollados en el momento de coordinación y exploración de la primera etapa de implementación de la estrategia.

Primera sesión: Taller 1. Presentación de la investigación y sensibilización de docentes de la Licenciatura en Educación Inicial. La sesión inició con la determinación de las principales expectativas de los presentes y su articulación con las intenciones del trabajo a desarrollar. Se definieron, el contenido y los métodos de trabajo para cada momento, así como los roles del facilitador y de cada grupo de trabajo según la actividad planificada para los estudiantes. Se presentó a los docentes el diagnóstico realizado y sus resultados, así como la argumentación teórica de la investigación. Esto incluyó los elementos que sirven de sustento al modelo pedagógico propuesto, además de las relaciones que se establecen en él; luego, se procedió a debatir las reflexiones de los participantes en el taller sobre las ideas presentadas en torno a la investigación; se registraron las recomendaciones brindadas las que se sintetizan a continuación. Como resultado, se obtuvo el consenso de todos relativo a perfeccionar el proceso de formación ambiental de los estudiantes dada la responsabilidad que estos desempeñarán en la educación de los niños/niñas. Se mostraron interesados y agradecen la forma en la que se presentaron los elementos de política educativa del Ecuador que argumentan la importancia de considerar la dimensión ambiental en la formación profesional. Evidenciaron su disposición de participar y apoyar en las actividades que se les convocara en lo adelante.

Segunda Sesión: Taller 2. Presentación del modelo pedagógico y su estrategia de implementación en la Sede Macas. La sesión inició con la argumentación de la necesidad de la investigación ilustrada esta desde los resultados del diagnóstico inicial aplicado a estudiantes de la Licenciatura Educación Inicial. Los participantes apoyaron la idea y expusieron cómo la problemática encontrada se manifiesta también en las restantes carreras de la universidad. En un segundo momento se presentó la estructura y componentes del modelo, así como las acciones a desarrollar desde la estrategia; todo ello fundamentado en la normativa ambiental y educativa del Ecuador. Los profesores felicitaron la iniciativa se mostraron sensibilizados al punto que sugirieron gestionar para replicar esta experiencia en sus carreras.

Tercera Sesión: Taller 3. Presentación del resultado científico, bases teórico-metodológicas y necesidad de la investigación; mediante la técnica del focus group. El desarrollo de la sesión vía on-line mediante la plataforma zoom, facilitó el intercambio entre un grupo de especialistas con funciones y visión diversa del objeto de la investigación, lo que constituyó una experiencia interesante que permitió el enriquecimiento de los resultados.

Los criterios emitidos por los especialistas presentes en el taller sobre los resultados del diagnóstico coinciden en que el estudio fue amplio y diverso; plantearon además que, fundamentar el modelo pedagógico desde la pedagogía crítica y el constructivismo social, hace que la tesis sea consecuente con los sustentos pedagógicos del proceso educativo en el país. Hubo consenso en la importancia de esta experiencia y la necesidad de desarrollar otras para trabajar la formación ambiental de los futuros profesionales, lo que se corrobora en otras investigaciones como la realizada por (Adela et al., 2018). Los participantes sugirieron considerar las habilidades o competencias para el desarrollo de los procedimientos (saber hacer); resaltaron la necesidad de trabajar las actitudes y comportamientos de los jóvenes e involucrarles en la autoevaluación de sí mismos, para alcanzar las competencias para la sostenibilidad, cuestiones que coinciden con las planteadas por (Sengupta et al., 2020) en su obra que trata sobre las estrategias de enseñanza y aprendizaje para el desarrollo sostenible.

Como parte del momento de coordinación y exploración también se realizaron las reuniones metodológicas con profesores de las cátedras: Educación ambiental y desarrollo sostenible, Necesidades educativas especiales, Estimulación temprana y Práctica pre-profesional, en ellas se identificaron los núcleos de 
contenidos más idóneos para trabajar la formación ambiental de los estudiantes. En otra reunión metodológica se discutieron y acordaron los métodos y formas de organización a utilizar en las actividades de formación ambiental propuestas para desarrollar con los estudiantes, cuyos resultados se muestran en la Tabla 1. En el segundo momento denominado de desarrollo, se ejecutaron las actividades concebidas desde la etapa de planificación de la estrategia. En el caso de capacitación a los docentes, se realizó a través del entrenamiento desde el puesto de trabajo y a distancia, como resultado se desarrollaron cinco sesiones, con los objetivos y participantes que se precisan en la Tabla 2.

Tabla 1: Actividades de formación ambiental a desarrollar con estudiantes de sexto ciclo

\begin{tabular}{|c|c|c|c|}
\hline Actividad de formación ambiental & Componente & Métodos & $\begin{array}{l}\text { Formas de } \\
\text { organización }\end{array}$ \\
\hline $\begin{array}{l}\text { 1. Formación ambiental, reclamo profesional en el } \\
\text { siglo XXI. Un debate desde su basamento } \\
\text { teórico-metodológico }\end{array}$ & Docencia & $\begin{array}{l}\text { Clarificación de } \\
\text { valores }\end{array}$ & Conferencia \\
\hline $\begin{array}{l}\text { 2. La naturaleza como sujeto de derecho, papel de } \\
\text { la educación }\end{array}$ & Docencia & $\begin{array}{l}\text { Clarificación de } \\
\text { valores }\end{array}$ & Conferencia \\
\hline 3. Educación inclusiva en un medioambiente sano & Docencia & Debate en grupos & Seminario \\
\hline $\begin{array}{l}\text { 4. Desarrollo sostenible, debate desde la educación } \\
\text { ecuatoriana }\end{array}$ & Docencia & Debate en grupos & Seminario \\
\hline 5. Signos de alerta en edades tempranas & Docencia & Estudio de caso & Clase práctica \\
\hline 6. Aprendiendo con 4R & Docencia & $\begin{array}{l}\text { Clarificación de } \\
\text { valores }\end{array}$ & Clase práctica \\
\hline 7. Cómo educar a los niños/as en el medioambiente & $\begin{array}{l}\text { Docencia/ } \\
\text { investigación }\end{array}$ & $\begin{array}{l}\text { Investigación- } \\
\text { acción }\end{array}$ & $\begin{array}{l}\text { Clases en la práctica } \\
\text { pre-profesional }\end{array}$ \\
\hline $\begin{array}{l}\text { 8. Ensayo sobre las competencias y rol del docente } \\
\text { inclusivo incluida la dimensión ambiental }\end{array}$ & $\begin{array}{l}\text { Docencia/ } \\
\text { investigación }\end{array}$ & $\begin{array}{l}\text { Clarificación de } \\
\text { valores }\end{array}$ & Clase práctica \\
\hline $\begin{array}{l}\text { 9. Diagnóstico ambiental. Vulnerabilidades y riesgos } \\
\text { de los niños y las niñas }\end{array}$ & Investigación & $\begin{array}{l}\text { Investigación- } \\
\text { acción }\end{array}$ & Clase práctica \\
\hline $\begin{array}{l}\text { 10. Investigar en el medioambiente. (posibilitó el } \\
\text { desarrollo de tres artículos científicos) }\end{array}$ & Investigación & $\begin{array}{l}\text { Investigación- } \\
\text { acción }\end{array}$ & $\begin{array}{l}\text { Ejercicio de fin de } \\
\text { estudios }\end{array}$ \\
\hline 11. Dramatizados ambientales & Vinculación & Debate en grupos & Taller comunitario \\
\hline 12. Grupo "Punto ecológico" & Vinculación & $\begin{array}{l}\text { Proyecto de } \\
\text { acción operativa }\end{array}$ & $\begin{array}{l}\text { Excursiones, talleres y } \\
\text { charlas educativas }\end{array}$ \\
\hline
\end{tabular}

Tabla 2: Actividades de capacitación a docentes

\begin{tabular}{|l|l|l|}
\hline Actividad & Objetivo & Participantes \\
\hline Reunión metodológica 1 & $\begin{array}{l}\text { Determinar en las diferentes asignaturas, los } \\
\text { contenidos idóneos para trabajar la formación } \\
\text { ambiental de los estudiantes }\end{array}$ & $\begin{array}{l}\text { Profesores responsables de } \\
\text { cátedras de segundo a } \\
\text { séptimo ciclos }\end{array}$ \\
\hline Reunión metodológica 1 & $\begin{array}{l}\text { Determinar en las diferentes asignaturas, los } \\
\text { contenidos idóneos para trabajar la formación } \\
\text { ambiental de los estudiantes }\end{array}$ & $\begin{array}{l}\text { Profesores responsables de } \\
\text { cátedras de segundo a } \\
\text { séptimo ciclos }\end{array}$ \\
\hline Reunión metodológica 2 & $\begin{array}{l}\text { Acordar los métodos y formas de organización a } \\
\text { utilizar en las actividades de formación ambiental } \\
\text { para desarrollar con los estudiantes }\end{array}$ & $\begin{array}{l}\text { Profesores de cátedras de } \\
\text { segundo a séptimo ciclos }\end{array}$ \\
\hline $\begin{array}{l}\text { Conferencia: Compromisos } \\
\text { de la educación ambiental } \\
\text { con el desarrollo sostenible. }\end{array}$ & $\begin{array}{l}\text { Debatir con la comunidad académica los principales } \\
\text { retos de la educación ambiental frente a los } \\
\text { compromisos del país y la región con la Agenda 2030 }\end{array}$ & $\begin{array}{l}\text { Docentes y directivos de las } \\
\text { carreras que se desarrollan } \\
\text { en la sede Macas. }\end{array}$ \\
\hline $\begin{array}{l}\text { Taller de socialización: } \\
\text { Normativas ambientales } \\
\text { aplicadas al contexto } \\
\text { educativo. (on-line) }\end{array}$ & $\begin{array}{l}\text { Argumentar las bases legales que sustentan la } \\
\text { dimensión ambiental en la formación profesional y } \\
\text { que han de ser consideradas en el contexto educativo }\end{array}$ & $\begin{array}{l}\text { Docentes y directivos de la } \\
\text { carrera Educación inicial }\end{array}$ \\
\hline $\begin{array}{l}\text { Sesión científica: } \\
\text { Modelación de la formación } \\
\text { ambiental de estudiantes, } \\
\text { carrera de Educación inicial. } \\
\text { (on-line) }\end{array}$ & $\begin{array}{l}\text { Socializar entre la comunidad académica de la sede } \\
\text { Macas, las ideas esenciales de un Modelo } \\
\text { Pedagógico para la formación ambiental de los } \\
\text { estudiantes de la Licenciatura en Educación Inicial y } \\
\text { su estrategia de implementación }\end{array}$ & $\begin{array}{l}\text { Docentes y directivos de la } \\
\text { carrera Educación inicial }\end{array}$ \\
\hline
\end{tabular}


En relación a las actividades desarrolladas con los estudiantes como parte de la implementación de la estrategia, desde la práctica pre-profesional y la cátedra de Necesidades Educativas Especiales en el período evaluado se ejecutaron un total de 12 actividades de formación ambiental listadas con anterioridad en la tabla 1. El control y la evaluación de los resultados obtenidos, como se explicó en la metodología, se realizó con carácter procesal mediante un registro de incidencias a partir de los parámetros establecidos para evaluar la variable por sus indicadores en niveles bajo, medio y alto; utilizando una escala de bajo=0; medio $=1$; alto $=2$, los resultados de la ponderación grupal por actividad y el promedio general se recogen en la Tabla 3.

Tabla 3: Registro de incidencias para la evaluación procesal de la variable

\begin{tabular}{|c|c|c|c|c|c|c|c|c|c|c|c|c|c|c|}
\hline \multirow[t]{2}{*}{ Dimensiones } & \multirow[t]{2}{*}{ Indicadores } & \multicolumn{13}{|c|}{ Actividades de formación ambiental } \\
\hline & & 1 & 2 & 3 & 4 & 5 & 6 & 7 & 8 & 9 & 10 & 11 & 12 & Promedio \\
\hline \multirow[t]{4}{*}{ Cognitiva } & $\begin{array}{l}\text { Identificación del medio ambiente } \\
\text { como sistema }\end{array}$ & 0 & 0 & 0 & 0 & 1 & 1 & 0 & 1 & 1 & 1 & 1 & 1 & 0,58 \\
\hline & $\begin{array}{l}\text { Argumentación de la problemática } \\
\text { ambiental en Ecuador (causas, } \\
\text { consecuencias) }\end{array}$ & 0 & 0 & 1 & 1 & 1 & 1 & 1 & 2 & 2 & 1 & 2 & 2 & 1,17 \\
\hline & $\begin{array}{l}\text { Reflexiones en torno a la formación } \\
\text { ambiental para su formación } \\
\text { profesional }\end{array}$ & 1 & 1 & 1 & 1 & 1 & 1 & 2 & 2 & 2 & 2 & 2 & 2 & 1,50 \\
\hline & $\begin{array}{l}\text { Conocimiento sobre la normativa } \\
\text { medioambiental del Ecuador }\end{array}$ & 0 & 0 & 0 & 1 & 1 & 1 & 1 & 1 & 2 & 1 & 2 & 2 & 1,00 \\
\hline \multirow[t]{3}{*}{ Procedimental } & $\begin{array}{l}\text { Habilidad para identificar problemas } \\
\text { ambientales, causas y } \\
\text { consecuencias. }\end{array}$ & 0 & 0 & 0 & 0 & 1 & 1 & 1 & 1 & 1 & 2 & 2 & 2 & 0,92 \\
\hline & $\begin{array}{l}\text { Capacidad para planificar actividades } \\
\text { educativas ambientales }\end{array}$ & 0 & 0 & 1 & 1 & 1 & 1 & 1 & 1 & 1 & 2 & 2 & 2 & 1,08 \\
\hline & $\begin{array}{l}\text { Valoraciones críticas respecto a la } \\
\text { problemática ambiental }\end{array}$ & 1 & 1 & 1 & 1 & 1 & 1 & 2 & 2 & 2 & 1 & 2 & 2 & 1,42 \\
\hline \multirow[t]{3}{*}{ Actitudinal } & $\begin{array}{l}\text { Muestra sensibilidad ante los } \\
\text { problemas ambientales y sus causas. }\end{array}$ & 1 & 1 & 1 & 1 & 2 & 2 & 2 & 2 & 2 & 2 & 2 & 2 & 1,67 \\
\hline & $\begin{array}{l}\text { Disposición para actuar en prácticas } \\
\text { ambientales sostenibles }\end{array}$ & 1 & 1 & 1 & 2 & 2 & 2 & 2 & 2 & 1 & 2 & 2 & 2 & 1,67 \\
\hline & $\begin{array}{l}\text { Sistematización de acciones y } \\
\text { buenas prácticas en el contexto } \\
\text { educativo }\end{array}$ & 0 & 0 & 0 & 0 & 0 & 1 & 1 & 1 & 1 & 1 & 1 & 1 & 0,58 \\
\hline
\end{tabular}

Relacionado con la dimensión cognitiva, se pudo determinar que el indicador identificación del medioambiente como sistema, constituyó el menos desarrollado (PromG=0,58), independientemente de las diferentes vías implementadas para debatir con los estudiantes y que estos comprendieran las relaciones de sistema que se dan en el medioambiente, este es un elemento que se ha de continuar trabajando por la importancia que ello representa para la transformación de actitudes y comportamientos.

En la dimensión procedimental, los tres indicadores tuvieron un promedio general que tiende al valor medio (Ind1=0,92; Ind2=1,08; Ind3=1,42), lo que demuestra el comportamiento de los alumnos en correspondencia con la visión limitada que predomina sobre el medioambiente. Al argumentar las causas y consecuencias de los problemas ambientales los estudiantes se centraban en factores de orden económico en divorcio con elementos sociales, aspectos que fueron corroborados en otros estudios como el realizado por (Cóndor, 2018) y el de (Rusinko, 2010). Se desatacó como elemento positivo que las planificaciones de actividades pedagógicas a desarrollar en sus escuelas de práctica preprofesional en la medida que la experiencia fue avanzando, ganaron en logicidad e interrelación entre ellas y con los problemas ambientales del contexto.

Finalmente, entre los indicadores de la dimensión actitudinal, fueron la sensibilización con la problemática ambiental (PromG $=1,67$ ) y la disposición a participar (PromG $=1,67$ ) los que de manera más temprana mostraron una tendencia de crecimiento positivo, aspecto que coincide con los hallazgos de la investigación realizada por (Dada et al., 2017) en Nueva Zelanda. En su gran mayoría, los estudiantes desde el inicio mismo de la experiencia se mostraron motivados, sin embargo, fue la sistematización de acciones y buenas prácticas en el contexto educativo (PromG=0,58) lo más demorado pues esto lleva mayor tiempo de maduración y requiere de la formación de determinadas competencias que aún no son evidentes en la muestra seleccionada. 
Durante el proceso de intervención en la práctica de la estrategia, se observó, que, en las primeras conferencias, la mayoría de los estudiantes no mostraban una actitud positiva ante la búsqueda del conocimiento sobre el medio ambiente y las acciones para su preservación, comentaban no tener las actitudes y comportamientos adecuados con relación a las acciones de formación ambiental. En la medida que iba avanzando el curso fueron cambiando de actitud, comenzaron a traer resúmenes realizados por ellos, comentaban la importancia de buscar el conocimiento para poder resolver las tareas y se fue fomentando una actitud de intercambio y colaboración, consideraban que la manera en que se trataba el contenido desde las clases les resultaba interesante, diferente y novedoso; específicamente la introducción de cada tema a través de las tareas integradoras, donde desde el rol de estudiantes debían buscar, comprender y operar con el conocimiento necesario para su solución. Con el desarrollo del ciclo, se observaron más independientes en la realización de las actividades, se logró que estas siempre fueran ejecutadas por ellos, con la ayuda necesaria en algunos casos, incluyendo sus profesores y hasta sus familiares, al estar conscientes de sus dificultades, mostraban mayor interés en la realización de las tareas.

Un aspecto motivante para los estudiantes fue la obtención de información a partir de la utilización de fuentes humanas (alumnos de otros cursos, docentes de educación inicial de los Centros de prácticas preprofesionales, profesores de la carrera, padres de los niños, personas de la comunidad, etc.). Este elemento, como lo expresaron ellos mismos no había sido objeto de atención anteriormente en otras asignaturas de la carrera. Resaltaron la utilidad de la relación entre las actividades con la investigación y la vinculación con la sociedad, para lograr un futuro Licenciado en Educación Inicial que reconozca la importancia que tiene la formación ambiental en la vida profesional, aspectos que son reconocidos en la obra de (Davis, 2020) quien señala que los estudiantes debe ver los problemas y las soluciones a través de los lentes de la crítica, particularmente de sus ideas y prácticas cotidianas de desarrollo humano, y que desarrollen capacidades de investigación y transformación.

Otra de las vías para emitir juicios de valor respecto a los resultados de las actividades desarrolladas con los estudiantes fue la aplicación del método experimental con el preexperimento. Como dato de interés se deben precisar los antecedentes que tenían sobre las experiencias y aprendizajes recibidos desde la cátedra de medioambiente y desarrollo en el segundo ciclo. El instrumento aplicado en las pruebas de entrada y salida permitió obtener información relacionada con la identificación de conceptos sobre el medioambiente, el conocimiento de la legislación ambiental del Ecuador y los principales problemas ambientales que afectan su país; así como el estado de sus actitudes y conductas hacia la preservación del medioambiente.

Los resultados obtenidos de la prueba pedagógica de entrada, se contrastaron luego con los de la prueba pedagógica de salida, mediante una rúbrica de evaluación que de manera automática por el sistema EVEA, tuvo en cuenta tanto el resultado al que llegaron, como la lógica del proceso seguido. Para medir las dimensiones e indicadores definidos para la formación ambiental, según la rúbrica, se tuvo en cuenta el siguiente rango de valores para calificar cada parámetro: Alto (0.6 a 0.8); Medio (0.3 a 0.5); Bajo (0 a 0.2). Los resultados totales de la evaluación en base a 10 puntos, se calcularon sumando los valores obtenidos en cada dimensión e indicador contenido en las preguntas.

Se tomaron los datos recolectados de la prueba de entrada y la prueba de salida de los 20 estudiante del 6to ciclo que participaron en la investigación y se sometió la variable a un análisis del antes y el después. Los datos de los cálculos correspondientes, a la prueba estadística de rangos con signos de Wilcoxon para dos muestras relacionadas, constatan que la medida de la T observada (estadígrafo que se obtiene a partir de la suma de los rangos de signo menos frecuentes), fue $T=33$, inferior, en un número considerable de los casos a la $T$ de la tabla (valores críticos $T=52)$, para un nivel de significación $(\alpha=0,05)$, según la tabla A-8 de "Diseño experimental no paramétrico" (Triola, 2009), lo que permite rechazar la hipótesis nula y aceptar la alternativa.

Por lo anterior se señala que hay suficiente evidencia desde el punto de vista estadístico para sustentar la aseveración de que existe diferencia entre los puntajes relativos a las pruebas de rendimiento que miden las dimensiones de la formación ambiental de los educandos antes y después de la aplicación de la estrategia. La figura 1, muestra que del total de estudiantes que respondieron la prueba (20), el 90\% (18) transitaron a un nivel superior en cuanto a su formación ambiental en base a las dimensiones e indicadores medidos; no obstante, en las respuestas que requerían construcción de propuestas o análisis de casos, se pudieron corroborar algunas insuficiencias con indicadores complejos tal y como se explicó con el análisis de registro de incidencias. La principal debilidad en este asunto continúa siendo la concepción errada que poseen en torno al medioambiente. Los resultados que se aprecian a partir de la aplicación de los diferentes métodos evidencian un avance en el nivel de la formación ambiental, si bien resulta obvio que en este período no es posible alcanzar el máximo logro significativo al respecto, se considera que con la implementación total de la propuesta, con un carácter interdisciplinario se lograría alcanzar la formación ambiental deseada en los estudiantes de la Licenciatura en Educación Inicial para que actúen como agentes de cambio en los centros de Educación Inicial al educar a los niños y en su interacción con los demás agentes de la comunidad educativa. 


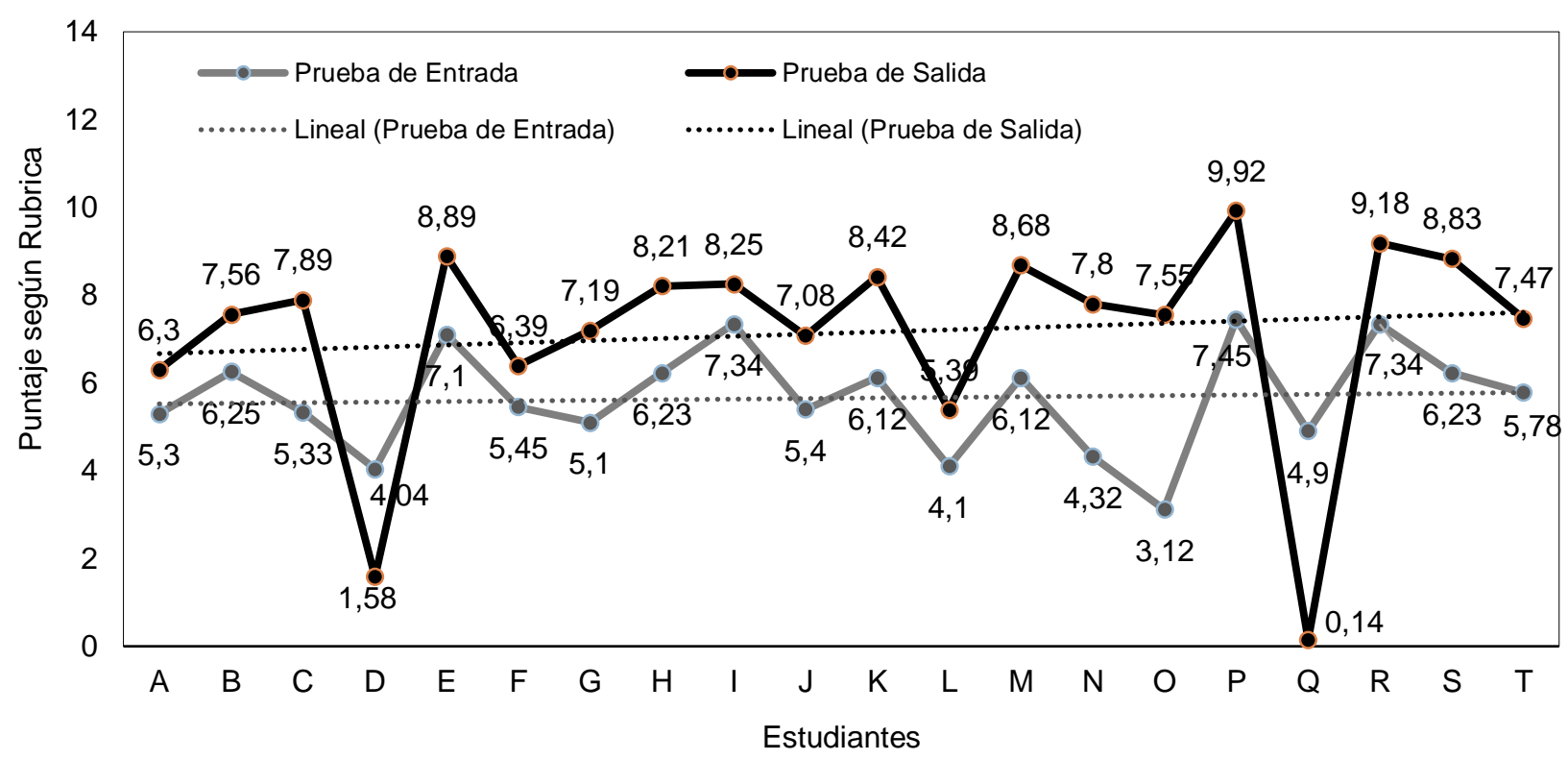

Fig. 1: Comparación de los puntajes relativos a las pruebas de rendimiento que miden las dimensiones de la formación ambiental de los educandos antes y después de la aplicación de la estrategia

\section{CONCLUSIONES}

De los resultados hallados y sus discusión es posible concluir que: 1) los referentes teóricos asumidos, hallan su expresión en el modelo pedagógico presentado como resultado científico, el que, según las bases de la pedagogía crítica y el constructivismo social aportó los elementos necesarios para la formación ambiental de los estudiantes con un enfoque holístico integrador; 2) la aplicación de la estrategia de implementación y la combinación de varios métodos empíricos permitió constatar la transformación positiva de la formación ambiental de los estudiantes en la Licenciatura en Educación Inicial de la Universidad Católica de Cuenca, Sede Macas observándose en el registro de incidencia que 5 indicadores tuvieron una tendencia hacia el nivel medio y 3 hacia el nivel alto; y en los resultados del preexperimento que un $90 \%$ logro transitar a un nivel superior; 3) si bien estos resultados son para un contexto específico, se observa que es posible promover la formación ambiental desde las universidades, con acciones que integren actividades que relacionen la docencia, la investigación y la vinculación orientadas al desarrollo de las dimensiones cognoscitiva, procedimental y actitudinal de la formación ambiental.

\section{REFERENCIAS}

Adela, D., Sukarno, S., y Indriayu, M., Integration of environmental education at the adiwiyata program recipient school in growing ecoliteracy of students, https://doi.org/10.2991/ictte-18.2018.11, Advances in Social Science, Education and Humanities Research, 262, 67-71 (2018)

Alcalá-Del-Olmo-Fernández, M. J., Rodríguez-Jiménez, C., Santos-Villalba, M. J., y Gómez-García, G., Educar para el desarrollo sostenible en el contexto universitario: un análisis bibliométrico, https://doi.org/10.4067/S071850062021000300085, Formacion Universitaria, 14(3), 85-94 (2021)

Álvarez, O., García, L. Á., Salvà, F., y Calvo, A., Variables influencing pre-service teacher training in education for sustainable development: A case study of two spanish universities, https://doi.org/10.3390/su11164412, Sustainability (Switzerland), 11(16), 4412 (2019)

Álvarez, O., Sureda, J., y Comas, R., Environmental education in pre-service teacher training: A literature review of existing evidence, https://doi.org/10.1515/jtes-2015-0006, Journal of Teacher Education for Sustainability, 17(1), 72-85 (2015)

Ardoin, N. M., Bowers, A. W., y Gaillard, E., Environmental education outcomes for conservation: A systematic review, https://doi.org/10.1016/j.biocon.2019.108224, Biological Conservation, 241(108224), 1-13 (2020)

ANE, Asamblea Nacional del Ecuador., Ley organica de educacion, http://www.conocimiento.gob.ec, Boletín Oficial del Estado, 106, 17158-17207 (2018)

Bertalanffy, L., General system theory foundations, development, applications, 1aㅡ Ed, 1-227, Braziller, ISBN: 9780807604533, New York, USA (1969)

Boca, G. D., y Saraçli, S., Environmental education and student's perception for sustainability, https://doi.org/10.3390/ su11061553, Sustainability (Switzerland), 11(6), 1-18 (2019) 
Carballo, M., y Guelmes, E. L., Algunas consideraciones acerca de las variables en las investigaciones que se desarrollan en educación, Revista Universidad y Sociedad, ISSN 2415-2897, 8(1), 140-150 (2016)

CEPAL., Horizontes 2030, La igualdad en el centro del desarrollo sostenible, https://repositorio.cepal.org (2016)

Cóndor, E. J., Dimensión ambiental en la formación profesional de los estudiantes de la facultad de educación de la Universidad Nacional de Huancavelica, https://doi.org/10.18800/educacion.201802.003, Educación, 27(53), 41-56 (2018)

Cuenca, J. M., Martín, M. J., y Estepa, J., Teacher training in heritage education: good practices for citizenship education, https://doi.org/10.1057/s41599-021-00745-6, Humanities and Social Sciences Communications, 8(1), 1-8 (2021)

Dada, D. O., Eames, C., y Calder, N., Impact of environmental education on beginning preservice teachers environmental literacy, https://doi.org/10.1017/aee.2017.27, Australian Journal of Environmental Education, 33(3), 201$222(2017)$

Davis, J. M., Creating change for people and planet: Education for sustainability approaches and strategies, https://doi.org/10.1016/B978-0-12-409548-9.12036-6, Encyclopedia of the World's Biomes, 52, $438-446$ (2020)

De-la-peña, G., y Vince, M.R., Acercamiento a la conceptualización de la educación ambiental para el desarrollo sostenible, Revista Cubana de Educación Superior, ISSN 0257-4314, 39(2), 1-15 (2019)

Flores, R., Medio ambiente y educación ambiental: representaciones sociales de los profesores en formación, Magis, ISBN 2027-1182,2(4), 401-413 (2010)

González, E., Environmental Education: a field in tension or in transition?, Environmental Education Research, ISSN 1469-5871, 12, 291-300 (2006)

Grishaeva, Y. M., Wagner, I. V., y otros 3 autores, Education for sustainable development today: a problem area for overcoming difficulties of pedagogical adaptation (on the example of a higher school), https://doi.org/10.18470/19921098-2018-3-159-166, South of Russia: Ecology, Development, 13(3), 159-166 (2018)

Isch, E., y Zambrano, A., Algunas experiencias significativas en Ecuador, En Educación para el desarrollo sostenible en la región andina, $1^{\mathrm{a}}$ Ed, OREALC/UNESCO, ISBN 978-956-8302-92-4, 11-69, Santiago, Chile (2008)

Kelle, U., y Bernhard, R., How to use mixed-methods and triangulation designs: An introduction to history education, https://doi.org/https://doi.org/10.18546/HERJ.16.1.02, History Education Research Journal, 16(1), 5-23 (2019)

Martínez-Iñiguez, J., Tobón, S., y Soto-Curiel, J., Ejes claves del modelo educativo socioformativo para la formación universitaria en el marco de la transformación hacia el desarrollo social sostenible, http://dx.doi.org/10.4067/S071850062021000100053, Formación Universitaria, 14(1), 53-66 (2021)

Mejía, M.A., Una educación ambiental desde la perspectiva cultural para la formación de profesores en ciencias naturales, https://doi.org/10.17151/luaz.2016.43.16, Revista Luna Azul, 43(43), 354-385 (2016)

Mejías, R., Loret de Mola, E., y Cardona, J. A., Modelo pedagógico de dimensión ambiental en la formación laboral investigativa de los profesionales en la universidad, Humanidades Médicas, ISSN 1727-8120, 18(2), 210 -227 (2018)

Merino, T., Logros y desafíos de la red universitaria REDMA: por la sostenibilidad ambiental de la educación superior en Cuba, https://doi.org/10.17151/luaz.2019.49.6, Revista Luna Azul, 49, 109-125 (2019)

Rusinko, C. A., Integrating sustainability in higher education: a generic matrix, https://doi.org/10.1108/14676371011058541, International Journal of Sustainability in Higher Education, 11(3), 250-259 (2010)

Sengupta, E., Blessinger, P., y Yamin, T. S., Introduction to teaching and learning strategies for sustainable development, https://doi.org/10.1108/S2055-364120200000019001, Innovations in Higher Education Teaching and Learning, 19, 3-14 (2020)

Sudan, D. C., y Zuin, V. G., Emancipation in environmental education: training at a Brazilian University, https://doi.org/10.17583/remie.2018.3203, Multidisciplinary Journal of Educational Research, 8(2), 205-230 (2018)

Triola, M. F., Estadística, 10ma , 1-904, Pearson Educación, ISBN 978-970-26-1287-2, México (2009)

UCACUE., Modelo Educativo, https://documentacion.ucacue.edu.ec/ (2016)

Zambrano, Á., Enfoque de la educación para el desarrollo sostenible en la formación docente en Ecuador, En Reflexiones sobre la formación y el trabajo docente en Ecuador y América Latina, $1^{\text {a }}$ Ed, Universidad Politécnica Salesiana, 100-114, ISBN: 978-9978-10-225-1, 100-114, Cuenca, Ecuador (2015) 